\title{
Performance Analysis of Vertical handoff metrics using Variance based algorithms
}

\author{
Abhijit Bijwe ${ }^{1}$, Dr. C.G.Dethe ${ }^{2}$ \\ Dept. of Electronics \& Communication Engineering, Priyadarshini Institute of Engineering \& Technology, Nagpur ${ }^{1}$ \\ Director, UGC-Academic staff College, RTMNU, Nagpur ${ }^{2}$
}

\begin{abstract}
Vertical handover is the evolving concept in $4 \mathrm{G}$ for seamless communication between heterogeneous networks. In this paper, our main objective is to analyze handover between two WLAN, two Wimax, two UMTS networks. The vertical handover decision is taken based on the variance based algorithm, which calculates the variance of parameters such as delay, jitter, bandwidth and packet loss for various above mentioned networks and the network with most of the parameters with minimum variance is selected. This algorithm is also compared with with other algorithms such as MEW (Multiplicative experiment weighting), SAW (Simple Additive Weighting), TOPOSIS (Technique for order preference by similarity to ideal solution) and GRA (Grey Relational Analysis). These algorithms are appropriate for different traffic classes. Simulation results for proposed variance based algorithm in Matlab is discussed and compared with other Multiple attribute decision making algorithm basis of bandwidth; jitter, delay etc. are discussed in the paper. It can be seen that the proposed variance algorithm gives less packet delay than all the algorithms, Jitter is also is least than all the other algorithms.
\end{abstract}

Keywords: SAW, TOPOSIS, GRA, MEW.

\section{INTRODUCTION}

In today's scenario, we are having access to various wireless networks such as WLAN, UMTS, Wimax etc. so there is need for switching from one wireless network to other for better services. Vertical handoff can be used for load balancing to get high data rate etc. mechanism can support such switching and maintain the connectivity from one network to another. For this mobile devices with multiple interface card which may help in connecting to different access networks. This is called to vertical handoff which is different from horizontal handoff where the MN moves from one BS to another BS in the same network i.e. homogeneous network. The multiple interface card in mobile node can choose the access links such as wireless local area network (WLAN) IEEE 802.11, worldwide inter-operability for microwave access (Wimax) IEEE 802.16, UMTS network. There should be seamless transfer to the link which is appropriate as per the requirement e.g. for video session high data rate required. Seamless vertical handoff between heterogeneous network is expected.

This can be done by using IEEE 802.21 standard (Media Independent handover) which enables vertical handoff. IEEE 802.21 creates frame work for the protocol needed for vertical handoff. Actual algorithms need to be implemented by the designer. The IEEE 802.21 standard gives link layer intelligence and other network information to higher layers in order to execute handover between different networks such as wi-fi and wi-max.The paper is organized as follows :

Section 2 explains the importance IEEE 802.21 and how it can be incorporated in ns-2

Section 3 explains about the multiple attribute decision making MADM algorithm such as TOPOSIS, MEW, SAW, GRA and how it is included at MAC layer so that the vertical handoff decision is taken depending on the traffic classes.

Section 4 gives the result and conclusion for the above algorithm on the basis of various parameters such as throughput jitter, dropping ratio, delay etc.

\section{IMPORTANCE OF MEDIA INDEPENDENT HANDOVER IEEE 802.21}

IEEE 802.21 provides framework for the protocol required for vertical handoff. It facilitates the seamless handover between heterogeneous network supporting different technologies. This standard provides link layer intelligence and related network information to upper layer to optimize vertical handoff[6].

IEEE 802.21 defines three types of services: Information services, Command services, Event services Information services provide multiple types of networks that exist in a given geographical area ,command services are used by higher layer to control physical, data link and logical link layers. Event services gives changes in state and transmission behavior of physical, data link, logical link layers.

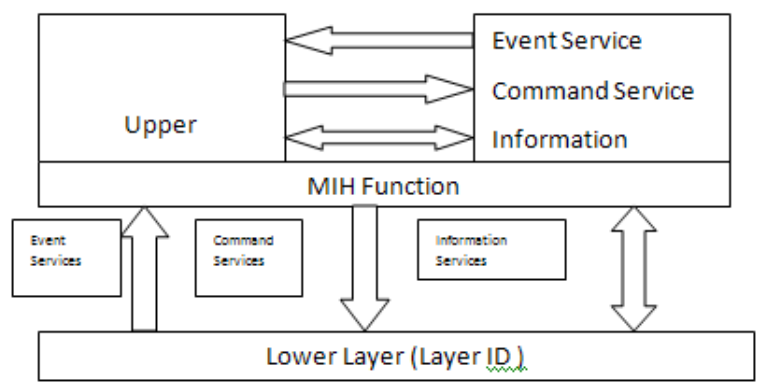

Fig 2.1 Media Independent handover 


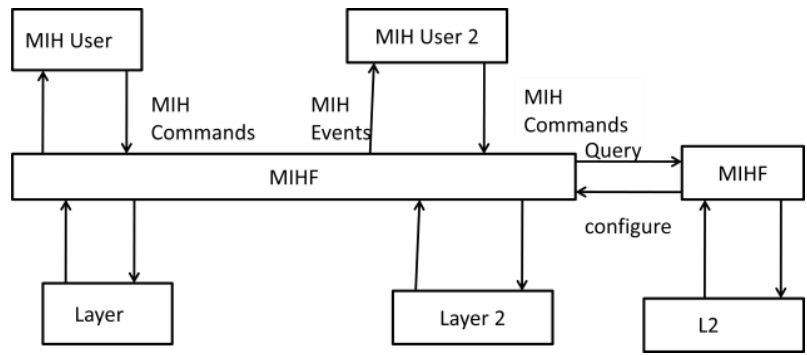

Fig 2.2 Media Independent handover frame work

III. PROPOSED VARIANCE BASED ALGORITHM Proposed algorithm is variance based algorithm which calculates the variance of parameters such as delay, jitter, bandwidth and packet loss for various above mentioned networks and the network with most of the parameters with minimum variance is selected. In our proposed algorithm, handoff metrics such as delay, bandwidth, jitter, packet loss etc are included.

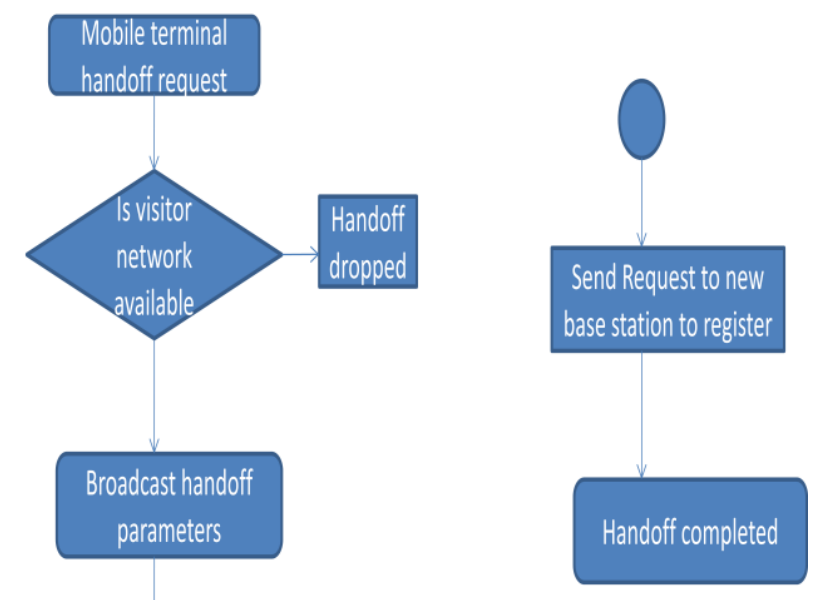

Calculate the variance of each

Parameters such as Delay, Jitter,

Bandwidth, packet loss etc

Network having most of the

minimum variance of the parameters

(eg: Delay, litter, BW etc) is selected

Fig 3.1 Flow chart of Proposed variance based algorithm Variance $=\frac{\sum(\boldsymbol{x}-\boldsymbol{\mu})^{2}}{\boldsymbol{N}}$ where $\mathrm{x}$ is any metrics such as delay, bandwidth, jitter etc and $\mu$ is its mean of a set of samples of the particular parameters. $\mathrm{N}$ is set of samples.

\section{OTHER MADM ALGORITHMS USED FOR VERTICAL HANDOVER}

SAW - simple additive weighting.[4]

Simple additive weighting(SAW) is the weighted sum method,where Score of each candidate network is calculated as below:

Score $_{\mathbf{S A W}}=\arg \operatorname{MAX} \sum_{\mathbf{j}=\mathbf{1}}^{\mathbf{N}}\left[\mathbf{W}_{\mathbf{j}} * \mathbf{N}_{\mathbf{i j}}\right]$
Where $\mathbf{N}_{\mathrm{ij}}=\mathbf{P}_{\mathrm{ij}} / \max \left(\mathbf{P}_{\mathrm{ij}}\right)$ for benefit parameters (eg: Bandwidth) $\mathbf{N}_{\mathbf{i j}}=\min \left(\mathbf{P}_{\mathbf{i j}}\right) / \mathbf{P}_{\mathbf{i j}}$ for cost parameters (eg:delay,Jitter). Pij can be any parameter such as Bandwidth, delay, jitter, packet loss etc. With i networks and $\mathrm{j}$ attributes.

The Score of each candidate network is found and the score with maximum value is selected.

TOPOSIS - Technique for order preference by similarity to ideal solution[4]

In this algorithm ideal solution is calculated. The selected candidate network will have shortest distance to ideal solution and will have longest distance from worst case solution.

To find the rank of network we this technique follows the step as below:

1. To construct normalized decision matrix consisting of various attributes such as Bandwidth, delay, Jitter, Packet loss etc $\mathbf{N}_{\mathrm{ij}}=\mathbf{P}_{\mathrm{ij}} / \sqrt{ } \sum_{\boldsymbol{m}}^{\boldsymbol{i}=\mathbf{1}} \boldsymbol{P}_{\boldsymbol{i} \boldsymbol{j}}{ }^{2}$

2. To Compute weighted normalized matrix by $\mathbf{V}_{\mathrm{ij}}=\mathbf{N}_{\mathrm{ij}} * \mathbf{W}_{\mathbf{j}}$ 3. To find positive and negative ideal solution $\mathrm{A}^{+}$ $=\left\{\left(\max V_{i j} / j \in J\right), \quad\left(\min V_{i j} / j \in J^{\prime}\right)\right\}, \quad A^{-}=\left\{\left(\min V_{i j} / j \in J\right)\right.$, $\left.\left(\max \mathrm{V}_{\mathrm{ij}} / \mathrm{j} \in J^{\prime}\right)\right\}$ where $\mathrm{J}$ is a set of benefit parameters and $\mathrm{J}$ ' is a set of cost parameters

4. Calculate the separation distance between available networks and positive and negative ideal solutions.

$$
\mathrm{S}^{+}=\sqrt{\sum_{j \epsilon N}\left(V_{i j}-V_{j}^{+}\right)^{2}} \quad, \mathrm{~S}^{-}=\sqrt{\sum_{j \epsilon N}\left(V_{i j}-V_{j}^{-}\right)^{2}}
$$

5. Compute the closeness to ideal solution $\mathrm{C}_{\mathrm{i}}=\mathrm{S}^{-} /\left(\mathrm{S}^{+}+\mathrm{S}^{-}\right)$

$$
\text { Score }_{\text {TOPSIS }}=\arg \max _{i \in M} \mathrm{C}_{\mathrm{i}}
$$

GRA(Grey relational Analysis)-

It is a technique of grey systems theory(GST) invented by Professor ju long Deng. Grey lies between black(no information) and white(full information) which means partial information. It is suitable for unascertained problems with poor information. GRA gives the similarity between the candidate network and best ideal network [4].Here, grey relational coefficient(GRC) is calculated, before that normalization of data and defining ideal sequence. Normalization of data is done for three situations larger the better, smaller the better, nominal the best.

$\mathrm{N}_{\mathrm{ij}}=\mathrm{P}_{\mathrm{ij}}-\min \left(\mathrm{P}_{\mathrm{ij}}\right) /\left(\max \left(\mathrm{P}_{\mathrm{ij}}\right)-\min \left(\mathrm{P}_{\mathrm{ij}}\right)\right)$

$\mathrm{N}_{\mathrm{ij}}=\max \left(\mathrm{P}_{\mathrm{ij}}\right)-\mathrm{P}_{\mathrm{ij}} /\left(\max \left(\mathrm{P}_{\mathrm{ij}}\right)-\min \left(\mathrm{P}_{\mathrm{ij}}\right)\right)$

$\mathrm{N}_{\mathrm{ij}}=1-\left|\mathrm{P}_{\mathrm{ij}}-\mathrm{m}_{\mathrm{j}}\right| / \max \left\{\max \left(\mathrm{P}_{\mathrm{ij}}\right)-\mathrm{m}_{\mathrm{j}}, \mathrm{mj}-\min \left(\mathrm{P}_{\mathrm{ij}}\right)\right\}$

$\mathrm{mj}$ is the largest value in the nominal the best situations.

$\Delta_{i}=\left|\mathrm{P}_{0 \mathrm{j}}-\mathrm{N}_{\mathrm{ij}}\right| \quad, \Delta_{\max }=\boldsymbol{m a x}_{\boldsymbol{i} \in \boldsymbol{M}, \boldsymbol{j} \in N} \Delta_{i}$,

$\Delta_{\text {min }}=\boldsymbol{m i n}_{\boldsymbol{i} \in \boldsymbol{M}, \boldsymbol{j} \in \boldsymbol{N}} \Delta_{i}$

$\mathrm{GRC}_{\mathrm{i}}=1 / \mathrm{m} \sum_{j=1}^{m}\left(\left(\Delta_{\max }+\Delta_{\min /\left(\Delta_{i}\right.}+\Delta_{\max }\right)\right)$

Score $_{\mathrm{GRA}}=\arg \max _{i \in M} \mathrm{GRC}_{\mathrm{i}}$

MEW -Multiplicative Exponent Weighting[4]

is the weighted product of attributes method,where SCORE $_{\text {MEW }}$ is calculated by the best values of attributes in networks.

$\mathrm{SCORE}_{\mathrm{MEW}}=\arg \max _{i \in M} \prod_{\mathbf{j}=\mathbf{1}}^{N}\left[\mathbf{N}_{\mathbf{i j}} \mathbf{w}_{\mathbf{j}}\right]$

The best algorithm with maximum score is selected for taking vertical handoff decision. 
International Journal of Advanced Research in Computer and Communication Engineering Vol. 4, Issue 8, August 2015

\section{RESULTS \& DISCUSSION}

Performance evaluation of algorithms are done using Matlab simulation, which considers three types of network with two each of the type .The networks considered are UMTS, WLAN, Wimax .

The Range of value for various parameters are as follows : Bandwidth for UMTS network 0.1-2Mbps, Packet delay for UMTS network 25-50ms, Jitter for UMTS network 5$10 \mathrm{~ms}$. Bandwidth for WLAN network 1-54Mbps, Packet delay for WLAN network 100-150ms, Jitter for WLAN network 10-20ms. Bandwidth for Wimax network 1$60 \mathrm{Mbps}$, Packet delay for Wimax network 60-100ms, Jitter for Wimax network 3-10ms.

Here, we consider three cases as follows:

1) All Parameters are given equal weights

2) Delay, jitter are given $70 \%$ weights which suits voice connections

3) Bandwidth is given $70 \%$ weights which suits data connections. These cases are applicable to the algorithms where weights are used.

In variance based algorithm, which calculates variance of each parameter and the network having at least two or more than two parameters with minimum variance is selected. In this algorithm weights are not assigned to the parameters.

In Proposed algorithm 10 iterations are taken i.e. handoffs are taken and the network selected in each iteration is also shown in the figure below .Here, 1,2,3,4,5,6 represents UMTS1,UMTS2,WLAN1,WLAN2,Wimax1, Wimax2 respectively

In the Simulation, Comparative analysis of various algorithms such as SAW, TOPSIS, MEW, GRA, Variance based algorithms. Various graphs have been plotted on

1.Packet Delay Vs Number of Handoffs

2.Jitter Vs Number of Handoffs.

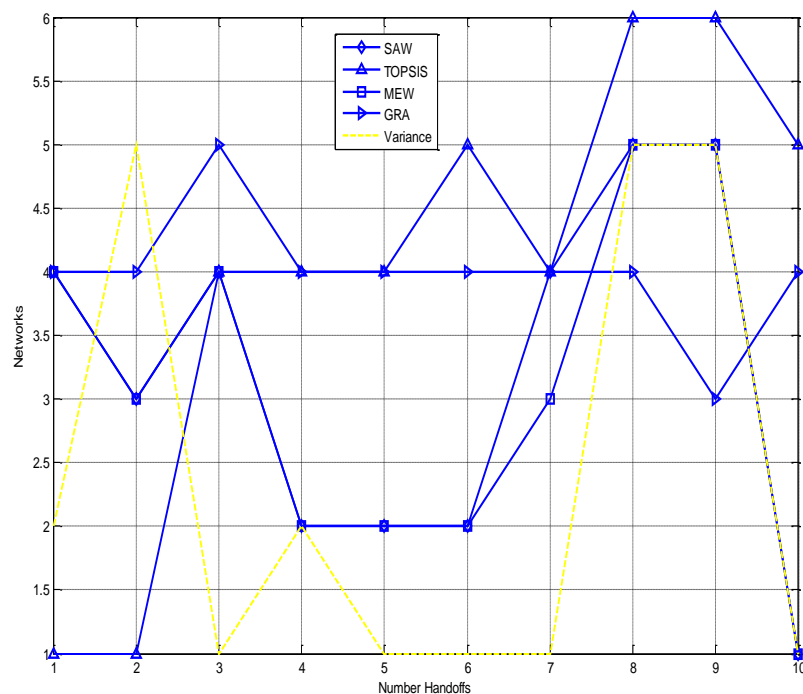

Fig 5.1 Network selection by the various algorithms

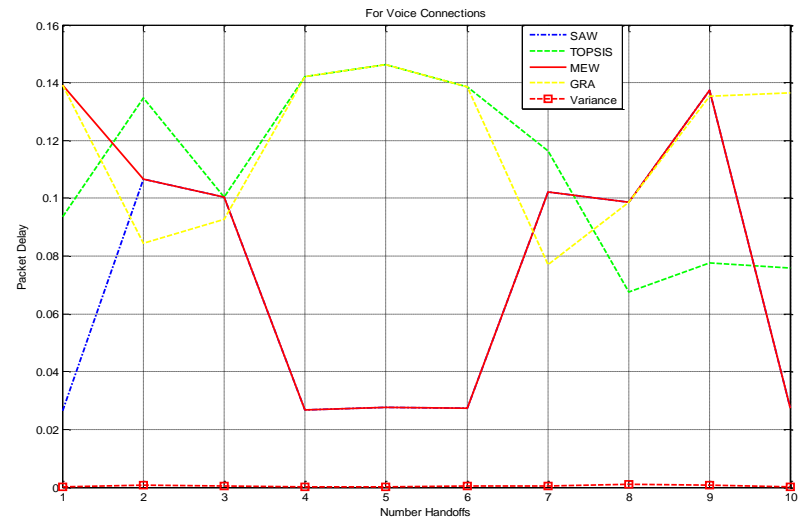

Fig 5.2 Packet delay vs number of handoffs for various algorithms

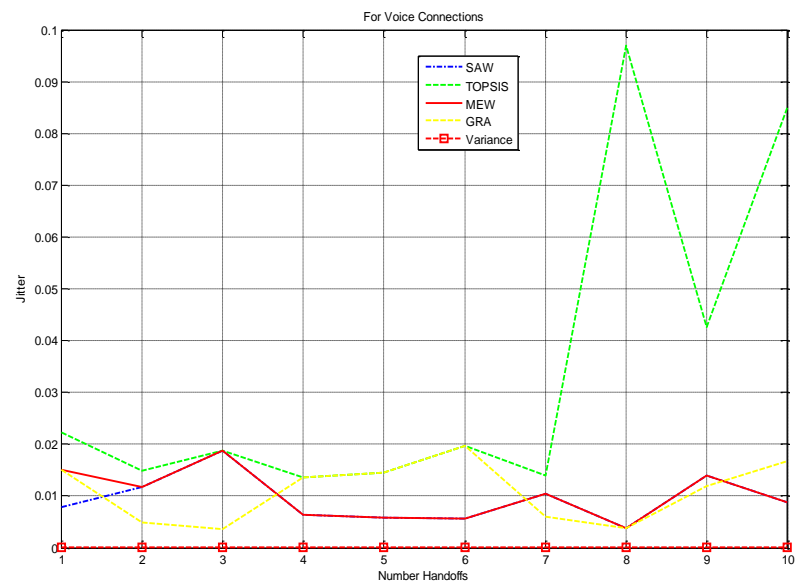

Fig 5.3 Jitter vs number of handoffs for various algorithms

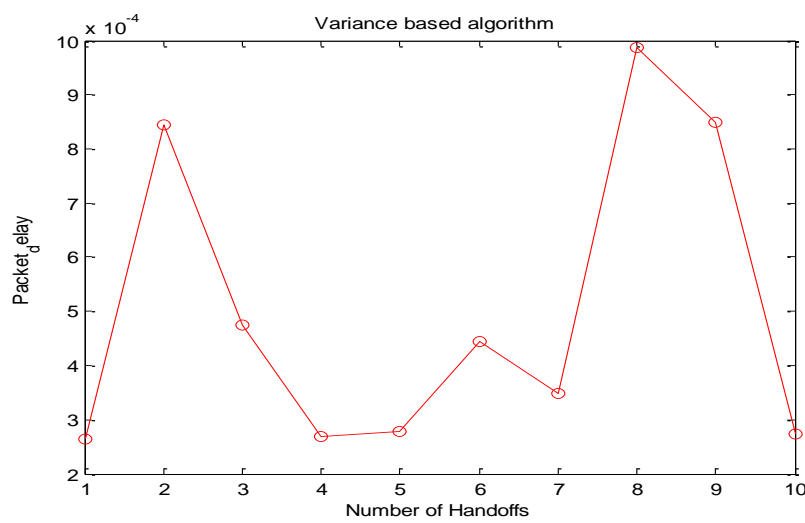

Fig 5.4 Packet delay vs number of handoffs for variance based algorithms

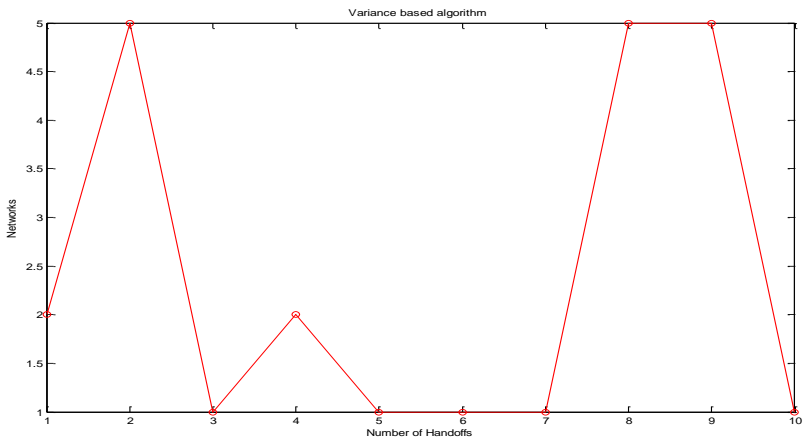

Fig 5.5 Network selection for variance based algorithms 


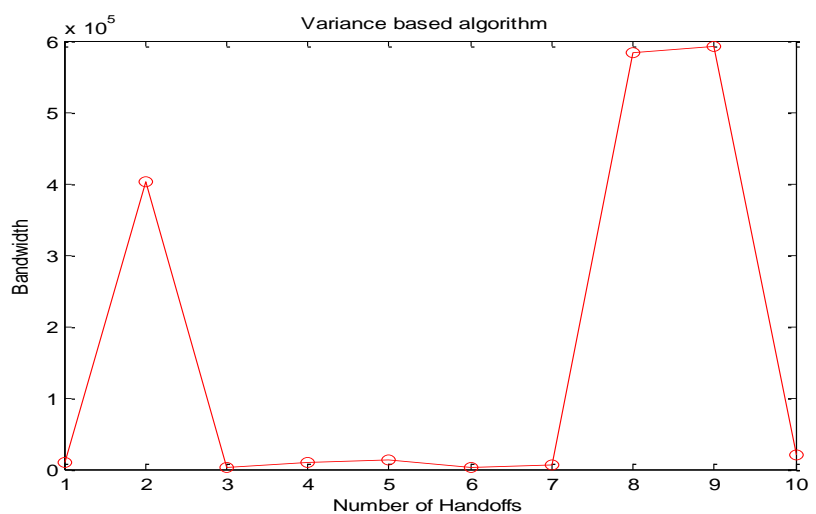

Fig 5.6 Bandwidth vs Number of handoffs for variance based algorithms

\section{CONCLUSION}

It is quite evident from above discussion that, the proposed algorithm has the lowest packet delay than MEW, SAW, GRA, TOPSIS algorithms. Also, it is observed that the proposed algorithm offers least jitter than any other above mentioned algorithms. These two observations makes the proposed algorithm best suited for voice connections. However, the observations obtained are from the simulation results. Hence, it is recommended that for practical scenarios the proposed algorithm can be utilized to have better voice communication.

\section{REFERENCES}

[1] T. S. Rappport, Wireless Communications:Principles and Practice. Prentice Hall, July 1999.

[2] S. Mohanty and I. F. Akyildiz, "A cross-layer(Layer $2+3$ ) handover management protocol for next-generation wireless systems," IEEE Trans. Mobile Computing, vol. 5, pp. 1347-1360, Oct. 2006.

[3] Yu-Chang Chen *, Ja-Hsing Hsia, Yi-Ju Liao, "Advanced seamless vertical handoff architecture for WiMAX and WiFi heterogeneous networks with QoS guarantees," Elsevier,2009, Pp.281-293

[4] Enrique Stevens-Navarro and Vincent W.S. Wong "Comparison between Vertical Handoff Decision Algorithms for Heterogeneous Wireless Networks",in :IEEE 0-7803-9392-9/06 2006 Pp.947-951

[5] Xiaohuan Yan,.Ahmet S, ekercio glu,Sathyanarayan "A Survey of vertical Decision algorithms in fourth generation Heterogeneous networks"Elsevier,2010, Pp.1848-1863

[6] HugoMarques, Jos'e Ribeiro,1 PauloMarques, and Jonathan Rodriguez, "Simulation of 802.21 Handovers Using ns-2," Hindawi Publishing Corporation Journal of Computer Systems, Networks, and Communications Volume 2010, Article ID 794749, 11 pages

[7] N. Nasser, S. Guizani, and E. Al-Masri. Middleware vertical handover manager: A neural network-based solution. In Proceedings of the 2007 IEEE International Conference on Communications (ICC'07), pages 5671\{5676, Glasgow, Scotland, June 2007.

[8] K. Pahlavan, P. Krishnamurthy, A. Hatami, M. Ylianttila, J. P. Makela, R. Pichna, and J. Vallstron. Handover in hybrid mobile data networks. IEEE Personal Communications, 7(2):34-47, 2000. 